\title{
Onuamental Horticulture
} REVISTA BRASILEIRA DE HORTICULTURA ORNAMENTAL

\begin{tabular}{|c|c|c|c|c|c|c|}
\hline Orn. Hortic. & Campinas-SP & v.22 & n.2 & p. 123-240 & May-Aug & 2016 \\
\hline
\end{tabular}

Sociedade Brasileira de Floricultura e Plantas Ornamentais 


\section{Ornamental Horticulture}

\section{Revista Brasileira de Horticultura Ornamental}

ISSN: 2447-536X

A publication of the Brazilian Society of Floriculture and Ornamental Plants

Publicação da Sociedade Brasileira de Floricultura e Plantas Ornamentais - SPFPO www.sbfpo.com.br

\section{EDITORIAL COMMITTEE}

Comissão editorial

EDITOR-IN-CHIEF | Editor Chefe

Dr. Gláucia Moraes Dias

ASSOCIATE EDITOR | Editor Associado

Dr. Patrícia Duarte de Oliveira Paiva

ASSISTANT EDITOR | Editor Assistente

Dr. Armando Reis Tavares

AREA EDITORS $\mid$ Editores de Área

Botany (Botânica):

Dr. Carmen Silvia Zickel | Universidade Federal Rural de Pernambuco (UFRPE), Recife-PE, Brazil

Biotechnology (Biotecnologia):

Dr. Fernanda Carlota Nery | Universidade de São João del Rei (UFSJ), São João del Rei-MG, Brazil

Economy \& Commercialization (Economia e Comercialização):

Dr. Emilson Caputo Delfino Silva | University of Alberta (UA), Edmonton, Canadá

Dr. José Edson Lara | Faculdades Pedro Leopoldo, Belo Horizonte-MG, Brazil

Landscaping (Paisagismo):

Dr. Maria Esmeralda Soares Payão Demattê | Universidade Estadual Paulista (UNESP), Jaboticabal-SP, Brazil

Dr. Daniella Nogueira Noraes Carneiro | Universidade Federal do Paraná (UFPR) Curitiba-PR, Brazil

Plant Diseases and Pests (Doenças e pragas):

Dr. Maria Amélia Vaz Alexandre | Instituto Biológico (IB), São Paulo-SP, Brazil

Dr. Paulo Sérgio Torres Brioso: Universidade Federal Rural do Rio de Janeiro (UFRRJ), Seropedica-RJ

Plant Physiology (Fisiologia):

Dr. Ana Maria Mapeli | Universidade Federal do Oeste da Bahia (UFOB), Barreiras-BA, Brazil

Dr. Fernando Luiz Finger | Universidade Federal de Viçosa (UFV), Viçosa-MG, Brazil

Postharvest (Pós-colheita):

Dr. Bruno T. Faveiro | University of Copenhagen, Copenhagen, Denmark

Dr. Ricardo Alfredo Kluge | Universidade de São Paulo (ESALQ), Piracicaba-SP, Brazil

Plant Propagation e Plant nutrition (Propagação, Nutrição):

Dr. Petterson Baptista da Luz | Universidade Estadual do Mato Grosso (UNEMAT), Cáceres-MT, Brazil

Dr. Leandro Flávio Carneiro | Universidade de Goiás (UFG), Cáceres-MT, Brazil

Plant Breeding, New Crops (Melhoramento, Novas culturas):

Dr. Alejandro Salvio Escandon | Istituto Nacional de Tecnologia Agropecuaria, Buenos Aires, Argentina

Dr. Mailson Monteiro do Rego | Universidade Federal da Paraiba (UFPB), Areia-PB, Brazil

Plant Science (Fitotecnia):

Dr. Gilmar Shafer | Universidade Federal do Rio Grande do Sul (UFRGS), Porto Alegre-RS, Brazil

Dr. Márkilla Zunete Beckmann-Cavalcante| Universidade Federal do Vale do São Francisco (UFVSF), Petrolina-PE, Brazil

BOARD - SPFPO

Diretoria - SPFPO

President (Presidente): Dr. Patrícia Duarte de Oliveira Paiva

Vice-president (Vice-Presidente): Dr. Fernando Luiz Finger

1st. Secretary ( $1^{\circ}$ Secretário): Dr. Marilia Andrade Lessa

2nd. Secretary (2 ${ }^{\circ}$ Secretário $)$ : Dr. Elka Fabiana Aparecida Almeida

1st. Tresaurer $\left(1^{\circ}\right.$ Tesoureiro): Dr. Simone Novaes Reis

2nd. Tresaurer $\left(2^{\circ}\right.$ Tesoureiro): Dr. Petterson Baptista da Luz

Executive-Secretary (Secretário-Executivo): Dr. Gláucia Moraes Dias

\section{Address (Endereço):}

Centro de Engenharia e Automação - Instituto Agronômico (IAC)

Av. Barão de Itapura, 1.481, Caixa Postal 28, 13012-970, Campinas, São Paulo.

e-mails: editor.ornamentalhorticulture@gmail.com or presidentesbfpo@gmail.com

Front cover (Capa) and Technical processing (Diagramação): Priscila Simionato Belavenute Front cover photo (Foto da capa): Michele Valquíria dos Reis 
Ornamental Horticulture

Revista Brasileira de Horticultura Ornamental

Sociedade Brasileira de Floricultora e Plantas Ornamentais, 1995

v.22, n.02, 2016

ISSN: 2447-536X

1. Horticultura. 2. Floricultura, 3. Plantas Ornamentais,

4. Paisagismo. 5. Jardins. I. Sociedade de Floricultura e Plantas Ornamentais

Indexação: AGRICOLA

AGRIS

AGROBASE

BINAGRI

CABI

CAB ABSTRACTS

LATINDEX

PERI

PERIODICA 


\section{SOCIEDADE BRASILEIRA DE FLORICULTURA E PLANTAS ORNAMENTAIS}

ORNAMENTAL HORTICULTURE

(Revista Brasileira de Horticultura Ornamental)

v.22, n.2, May-Ago 2016

Viabilidade econômica da produção de crisântemos em vaso no município de Atibaia-SP

CaioShigueakiShiroto, Narah VieiraPeres, OmarJorgeSabbag....

Quantitative inventory and analysis of the green areas in Lavras-MG and index evolution

Karina Volpi Furtini Boldrin, Cecília Souza Gontijo Garcia, Patrícia Duarte de Oliveira Paiva, Larissa Martiniano de Carvalho

Lisianthus cultivation using differentiated light transmission nets

Julia MartelladeAlmeida, CristianeCalaboni,PauloHercílio Viegas Rodrigues.

Flower opening and vase life of gladiolus cultivars: the sensitivity to ethylene and the carbohydrate content

Lucas CavalcantedaCosta, FernandoLuizFinger.

Atributos desejáveis para gramados a serem cultivados sob condições brasileiras: uma proposta

Francisco H. Dübbern de Souza, Marcos Rafael Gusmão, Frederico de Pina Matta, Ana Cecília Ribeiro de Castro, Andréa Mittelmann, Alessandra Pereira Fávero, Liana Jank.

Armazenamento úmido e seco de rosas cortadas

Gláucia Moraes Dias, JoséMaria Monteiro Sigrist, Patricia Cia, Sylvio Luís Honório. $166-171$

Parque Ecológico Quedas do Rio Bonito: Percepções da frequência de visita

Mariel de Carvalho Rafael Salgado, Paulo Henrique Fernandes Figueiredo, Karina Volpi Furtini Boldrin,

Rafael de Brito Sousa, Patricia Duarte de Oliveira Paiva.

Heliconia 'Golden Torch' postharvest: stem ends cutting and renewing vase water benefits

Wladiney Rodrigues Folha, Rafaela Ribeiro de Souza, Genilda Canuto Amaral, Alcilane Arnaldo Silva,

Jullyanna Nair de Carvalho, Márkilla Zunete Beckmann Cavalcante.

Maturação, temperatura e quebra de dormência na germinação de sementes de sempre-vivas Andréa dos Santos Oliveira, Maria laene Moreira de Carvalho, Cláudio das Neves

Vieira Bárbara, Tanismare Tatiana de Almeida, Marcela Carlota Nery.

Salinity on the germination of seed and index of germination speed of three ornamental species Marcos Vieira Ferraz, Claudenir Facincani Franco, Gisele Sales Batista, Kathia Fernandes Lopes Pivetta

Desenvolvimento de alpínia sob diferentes telas de sombreamento e espaçamentos de cultivo Roseane Rodrigues de Souza, Ângela Maria Pereira Nascimento, Patrícia Duarte de Oliveira Paiva, Elka Fabiana Aparecida Almeida, Paulo Roberto Correa Landgraf 
Fertilizantes agrícolas aplicados via solução nutritiva em Cattleya labiata Lindl.

Rodrigo Thibes Hoshino, Guilherme Augusto Cito Alves, Renan Ribeiro Barzan, Gustavo Adolfo de Freitas Fregonezi,

RicARDo TADEU DE FARIA ....

Biometry of fruits and seeds, dormancy and substrates in seeds germination of Dypsis lutescens

Rogério Gomes PêGo, José Antonio SARAiva Grossi

Sulfuric acid on breaking dormancy seeds and on emergence and morphology of Canna edulis seedlings

Salinity in Rose Production

Michele Valquíria dos Reis, Júnia Rafael Mendonça Figueiredo, Renato Paiva, Diogo Pedrosa Correa Da Silva, Camila

Vitoria Nunes de Faria, Lauravaughn Rouhana 